\title{
Quality of Service Evaluation of SaaS Modeler (Cloudlet) Running on Virtual Cloud Computing Environment using CloudSim
}

\author{
Pradeep Singh Rawat \\ Computer Science \& Engg. \\ Department \\ Dehradun Institute of Technology \\ Dehradun, India
}

\author{
G. P. Saroha \\ Information Technology \\ Department \\ Dehradun Institute of Technology \\ Dehradun, India
}

\author{
Varun Barthwal \\ Information Technology \\ Department \\ H N B Garhwal University \\ Srinagar, India
}

\begin{abstract}
Internet based computation is the demand of present IT infrastructure. All computational operations are handled by the network of networks. Internet based computing i.e. cloud computing is the best alternative for handling the IT resources and use IT as a service. To increase the server utilization from $15 \%$ to $60 \%$ utility computing stands for reply. Best way to understand the functionality of Cloud Computing is cloud simulation tool. Cloud simulation tool provide the test bed to understand the association of cloud entity and event. Tool provides the sustainable, fault tolerant environment for experimental evaluation of cloud based application like social sites and scientific work flow. Using simulation tool we can find out the finish time taken by the SaaS modeler to run over the virtual machine using resource provisioning algorithm i.e. time shared and space shared at each level. We follow the basic layered architecture of Cloud Computing.
\end{abstract}

\section{General Terms}

CloudSim Toolkit, Virtualization, Cloudlet, Timeshared policy.

\section{Keywords}

Cloudsim, MIPS, gridlet, Virtual machine, Data center, Simulation, SaaS, PaaS, IaaS, VM.

\section{INTRODUCTION}

Cloud computing is the Internet based computing in which all computational operation is made to be performed over the cloud. We know that for resource management more cost need to be pay. So it is better to use the resources on rent basis rather than to buy our own resources. Each organization wants to make busy their employee for innovation and high quality resource utilization. Cloud Computing is basically increasing the utilization of IT infrastructure. Simplest definition of cloud computing is "To provide IT as a service" is called cloud computing. This is the part of distributed computation. The main component of IT is hardware, software (application, system) etc are provided as a service by the cloud Computing. While using cloud computing cloud vendors can provides the secure pool of resources which include the storage and computing server or blade server. It provides the massive distributed environment which may dynamic in nature. To control this type of distributed system we need to study some simulation Tool. Simulation tool which are used for distributed application based on object oriented programming. Simjava, Grdilet, Cloudsim, CloudAnalyst are the cloud simulation tool which provide the clue to us how to deploy application and what are the IT infrastructure requirements for the application. These tools follow the layered architecture i.e. user can add their own layer over the user code level. Simulation tool provides the prior information about cloud resources which are required for application deployment. We can use our own policy at data center level to share the MIPS of the physical processing element. Using simulation tool we can setup the different cloud configuration with internet characteristics. Processing power of the CPU to run their application is provisioned in time and space shared mode. We take an example of social networking application to deploy at different region with different internet characteristics, data center configuration.

\section{RELATED WORK}

Distributed system consist a collection of inter connected and virtualized computers that are dynamically provision and presented as one or more unified computing resources. Their service-level agreements established through negotiation between the service provider and consumers [1].The level on which computing services are offered to the consumer varies according to the abstraction level of the service. In the lowest level, Infrastructure as a Service (IaaS), services are supplied in the form of hardware where consumers deploy virtual machines, software platforms to support their applications. An example of an IaaS service is Amazon EC2 [7].In the next level, Cloud consumers do not have to handle virtual machines. Instead, a software platform for hosting applications (typically, web applications) is already installed in an infrastructure and offered to consumers. Then, consumers use the platform to develop their specific application. This strategy is known as Platform as a Service (PaaS) Examples of this case are Google App Engine [8] and Aneka. Finally, in Software as a Service (SaaS), an application is offered to consumers, which do not have to handle virtual machines and software platforms that host the application. Repeatable and controlled experiments on any of these levels require the use of other experimentation methodologies than real execution in a real platform. Simulation is one of such alternative and this is the focus of this work. There are many simulation techniques to investigate behavior of large scale distributed systems, as well as tools to support the research work. Some of these simulators are GridSim [2], Micro Grid [3], GangSim [12], SimGrid [4] and CloudSim 
[5]. While the first three focus on Grid computing systems, CloudSim is, for the best of our knowledge, the only simulation framework for studying Cloud computing systems. Nevertheless, grid simulators have been used to evaluate costs of executing distributed applications deployed in Cloud infrastructures [8] [9]. GridSim toolkit was developed to address the problem of performance evaluation of real large scaled distributed environments (typically Grid systems but it also supports simulation of P2P networks) in a repeatable and controlled manner. GridSim toolkit is a Java-based simulation toolkit that supports modeling and simulation of heterogeneous Grid resources, users spread across multiple organizations with their own policies for scheduling applications.

It supports multiple application models and Provides primitives for creation of application tasks, mapping of tasks to resources, and managing of tasks and resources. CloudSim enables seamless modeling, simulation, and experimenting on Cloud computing infrastructures. It is a self-contained platform that can be used to model datacenters, service brokers, and scheduling and allocation policies of large scale Cloud platforms. It provides a virtualization engine with extensive features for modeling life-cycle management of virtual machines in a data center, including policies for provisioning of virtual machines to hosts, scheduling of resources of hosts among virtual machines, scheduling of tasks in virtual machines, and modeling of costs incurring in such operations. CloudSim framework is built on top of GridSim toolkit. CloudSim allows simulation of scenarios modeling IaaS, PaaS, and SaaS, because it offers basic components such as Hosts, Virtual Machines, and applications that model the three types of services. Cloud Analyst is built directly on top of CloudSim toolkit, leveraging the features of the original framework and extending some of the capabilities of CloudSim. Cloudsim design and features are presented in the next section.

\section{CLOUDSIM}

Cloud user can deploy the large scale application over the real cloud without taking any responsibility for resource management and resource provisioning. Cloudsim toolkit provides the modeling and simulation of cloud computing system and application provisioning policy implementation. We can model the cloud component using this simulation tool kit. Cloud main resource datacenter can be model and configured across the different time zone. Internet applications are accessed by users around the world. This Simulation tool provides the repeatable and controlled environment to setup our own virtual cloud computing environment with different cloud component properties. Using cloudsim toolkit we evaluate the performance of SaaS modeler on the basis of estimated finish time like social networking application. We get the simulation results for Cloudlet running over the cloud environment implemented over the cloudsim at user code level. These results are helpful in quality of service improvement. Finish time of Cloudlet run act as a performance evaluation parameter for cloud task or Cloudlet. Cloudsim Toolkit provides the flexibility to the user to implement his own resource provisioning policy. To construct the virtual cloud computing environment we use the layered architecture of cloudsim and implement the virtual cloud environment at user code level. We inherit the features of bottom layers of the tool.

\section{MODELING VM ALLOCATION}

One of the key aspects that make a Cloud computing infrastructure different from a Grid computing infrastructure is the massive deployment of virtualization tools and technologies. Hence, as against Grids, Clouds contain an extra layer (the virtualization layer) that acts as an execution, management, and hosting environment for application services. CloudSim implements the time-shared and space-shared provisioning policies. Here we use Timeshared policy for cloud resource provisioning as shown in figure 2 below. We use Timeshared policy to allocate the processing core to the virtual machine and Cloudlet task to the virtual machine. Cloudlet task uses the slice of MIPS assigned to the associated virtual machine.

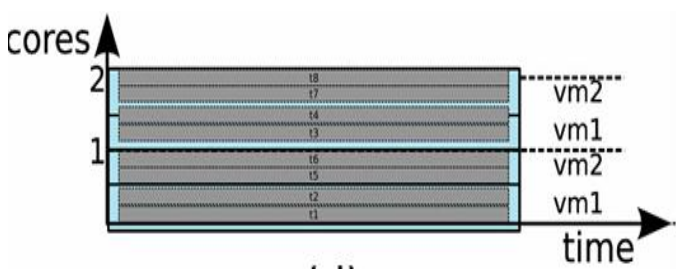

Figure 1: Time-shared provisioning for VMs and tasks

Timeshared policy forms the basis for allocating task units to processing core within a VM. Hence, during a VM lifetime all the tasks assigned to it are dynamically context switched during their lifecycle By using a time-shared policy, the estimated finish time of a Cloudlet managed by a VM is given by

$$
\text { Eft }(\mathrm{p})=\mathrm{ct}+\mathrm{rl} / \text { capacity } * \operatorname{cores}(\mathrm{p})
$$

Where eft(p) is the estimated finish time, ct is the current simulation time, and cores $(\mathrm{p})$ is the number of cores (processing elements) required by the Cloudlet, rl stands for cloudlet length remain to run over virtual machine. In time-shared mode, multiple Cloudlets (task units) can simultaneously multi-task within a VM. In this case, we compute the total processing capacity of Cloud host as

$$
\text { Capacity }=\sum_{i=1}^{n p} \operatorname{cap}(\mathrm{i}) / \max \left(\sum_{\mathrm{j}=1} \operatorname{cores}(\mathrm{j}), \mathrm{np}\right)
$$

Where cap(i) is processing strength of individual elements, $n p$ represents the number of processing elements.

\section{CLOUD COMPONENT DESCRIPTION}

Table 1. SaaS modeler properties

\begin{tabular}{|c|c|c|c|}
\hline Id & Length & $\begin{array}{c}\text { Input file } \\
\text { size(Byte) }\end{array}$ & $\begin{array}{c}\text { Output file } \\
\text { size(Byte) }\end{array}$ \\
\hline 10 & 4000 & 300 & 300 \\
\hline 20 & 2300 & 20 & 20 \\
\hline 25 & 1500 & 30 & 30 \\
\hline 30 & 800 & 10 & 10 \\
\hline
\end{tabular}

Above table 1 contain the details about properties of SaaS modeler which we want to deploy over the virtual machine in virtual cloud computing environment. It provides the information about the cloudlet id, cloudlet length, and size of 
input and output file in Byte. Cloudlet id is used to identify the cloudlet in a submitted cloudlet list. Cloudlet run time over the virtual cloud depends on strength of physical node at data center and cloudlet length.

Table 2. PaaS properties for Virtualization management

\begin{tabular}{|c|c|c|c|c|c|c|}
\hline Mips & Id & $\begin{array}{c}\text { Image } \\
\text { size } \\
\text { (MB) }\end{array}$ & $\begin{array}{c}\text { Vm } \\
\text { Memory } \\
(\mathbf{M B})\end{array}$ & $\begin{array}{c}\text { Band } \\
\text { width } \\
\text { (Mbps) }\end{array}$ & $\begin{array}{c}\text { Pes } \\
\text { No. }\end{array}$ & Vmm \\
\hline 100 & 1 & 10000 & 512 & 1000 & 1 & Xen \\
\hline 1000 & 4 & 10000 & 1024 & 100 & 2 & Xen \\
\hline 500 & 6 & 10000 & 256 & 1000 & 1 & Xen \\
\hline 200 & 7 & 10000 & 128 & 10 & 1 & Xen \\
\hline
\end{tabular}

Above table 2 include the details about application deployment configuration parameters which include the properties of virtual machine. It also includes the processing power of physical computing node which is assigned at the virtual machine level. Pes No. indicates the number of cores which MIPS power is shared at VM level to run the Cloudlet.

Table 3. IaaS properties for Cloud Simulation

\begin{tabular}{|c|c|c|c|}
\hline Id & $\begin{array}{c}\text { RAM } \\
\text { (MB) }\end{array}$ & Storage & $\begin{array}{c}\text { Band } \\
\text { width(Mbps) }\end{array}$ \\
\hline 13 & 1024 & 1000000 & 10000 \\
\hline 14 & 2048 & 1000000 & 1000 \\
\hline 15 & 512 & 1000000 & 10000 \\
\hline 16 & 256 & 1000000 & 100 \\
\hline
\end{tabular}

The entire information about storage, computing, network resource available at the data center at physical hardware level is displayed in Table 3. For better response time, we have to use stronger computing resources at infrastructure level. The resources available at this level put the limit on SaaS modeler requirement i.e. resources allocated at the VM level can't exceed this limit.

\section{A CASE STUDY}

\subsection{Simulation of SaaS Modeler Using Virtual Cloud Computing Environment}

Modeling and simulation of Cloud computing Environment in which we use the variable Cloud computing models. To perform performance analysis of Cloud Environment using different test cases i.e. Cloud simulation with one Cloud resource including Cloud component at three SaaS modeler properties with Id, length, file size for input and output operation. We get the Simulation results for SaaS Modeler running over the different virtual cloud computing environment. To run the run Cloudlet we use Timeshared and space shared policy at virtual machine level with in the data center. User can use their on policy for Cloud resource provisioning. We get the Quality of service measurement parameters. i.e. finish time of SaaS modeler. We use the event based simulator i.e. cloudsim hence it is not the way that event is processed according to the time but it is the way that time is passing according to the events. is based on Gridsim and Simjava so event features are extended from the java based simulator for utility computing. Construct the virtual cloud computing environment having cloud component properties as shown in the above section in tabular form.

\section{EXPERIMENTAL RESULTS FOR SAAS MODELER}

Table 4. Simulation results for Cloudlet (SaaS modeler) run

\begin{tabular}{|c|c|c|c|c|}
\hline $\begin{array}{c}\text { Cloulet } \\
\text { Id }\end{array}$ & Status & $\begin{array}{c}\text { Data center } \\
\text { Id }\end{array}$ & Vm Id & $\begin{array}{c}\text { Finish } \\
\text { Time }\end{array}$ \\
\hline 10 & success & 2 & 1 & 40 \\
\hline 20 & success & 2 & 4 & 2.3 \\
\hline 25 & success & 2 & 6 & 3 \\
\hline 30 & success & 2 & 7 & 4 \\
\hline
\end{tabular}

Table 4 shows the Results of experiment setup over the virtual cloud computing Environment. Cloud computing environment is setup at user code level in cloudsim following the layered architecture. We get the value of finish time of Cloudlet running over the virtual machine in timeshared mode. Cloudlet is submitted to the cloud broker or data center broker. This is the responsibly of cloud broker to assign the virtual machine to the cloudlet with optimal configuration. To run the cloudlet there are two choices to allocate the MIPS power either at VM level or host level. While running the cloudlet there may be set of events i.e. message passing among the cloud entities to run the application. Results depends on cloud component described in the table 1,2 and 3. We get the optimal configuration in case of row third. Minimum value of quality evaluation parameter indicate the exact values of cloud components to setup the cloud computing environment. Input data set for PaaS, and IaaS model which provide the optimal results of run time. These parameters set the infrastructure and platform for SaaS modeler run.

\section{CONCLUSION}

To study and analyze the cloud base application performance under the cloud computing environment, we setup our own virtual cloud computing environment and perform test to identify the performance of Cloudlet. Entire Application like facebook cannot be deployed without using real cloud. Task model is used to model application by Cloudlet. We get the value of execution time taken by the cloudlet to run over the virtual machine in a timeshared mode. Quality of service evaluation parameter value is strongly affected by the available storage, compute and network resources at infrastructure level. Analysis of heavy application is quite tedious, so we considered the user request as SaaS modeler. To test the performance of their provisioning and service delivery policies in a repeatable and controllable environment we extend the Cloudsim at user code level. We got the simulation results for the cloud task using cloud environment setup at user code level because we cannot deploy the entire cloud base application over virtual cloud environment. We get the simulation results for Cloudlet which holds the user request, user program running over the virtual 
cloud with optimal cloud configuration. Using our own resource provisioning policy at virtual machine level, we can improve the quality of service. Simulation results help us to fine tune the performance while deploying the application over the real cloud.

\section{REFERENCES}

[1] R. Buyya, C. S. Yeo, and S. Venugopal, "Market-Oriented Cloud Computing: Vision, Hype, and Reality for Delivering IT Services as Computing Utilities", Proceedings of the 10th IEEE International Conference on High Performance Computing and Communications (HPCC 2008, IEEE CS Press, Los Alamitos, CA, USA), Sept. 25-27, 2008, Dalian, China.

[2] R. Buyya, and M. Murshed, "GridSim" a toolkit for the modeling and simulation of distributed resource management and scheduling for Grid computing," Concurrency and Computation: Practice and Experience, vol. 14 , no. $13-15$, pp. 1175-1220, 2002.

[3] L. X. Song H, Jakobsen D, Bhagwan R, Zhang X, Taura K, A.Chien, "The Micro Grid: A scientific tool for modeling computational Grids," Proc. of the ACM/IEEE Super computing Conference, IEEE Computer Society, Nov. 2001.

[4] A. Legrand, L. Marchal, and H. Casanova, "Scheduling distributed applications: the SimGrid simulation framework," Proc. of the $3^{\text {rd }}$ IEEE/ACM International Symposium on Cluster Computing and the Grid (CC Grid 07), May 2001, pp. 138-145.

[5] R. Buyya, R. Ranjan, and R. N. Calheiros, "Modeling and Simulation of Scalable Cloud Computing Environments and the CloudSim Toolkit: Challenges and Opportunities," Proc. of the $7^{\text {th }}$ High Performance Computing and Simulation Conference (HPCS09), IEEE Computer Society, June 2009.
[6] J. Gustedt, E. Jeannot, and Martin Quinson, "Experimental methodologies for large-scale systems: a survey," Parallel Processing Letters, vol. 19, Sep. 2009, pp. 399-418.

[7] "Facebook," http://www.facebook.com.[9] "Amazon Elastic Compute Cloud (Amazon EC2),"http://aws.amazon.com/ec2/

[8] “Google App Engine,” http://code.google.com/appengine/

[9] E. Deelman, G. Singh, M. Livny, B. Berriman, and J. Good, "Thecost of doing science on the Cloud: the Montage example," Proc. of the 2008 ACM/IEEE Conference on Supercomputing, IEEE, Nov. 2008.

[10] M. Assunção, A. di Costanzo, and R. Buyya, "Evaluating the Cost-Benefit of Using Cloud Computing to Extend the Capacity of Clusters", Proc. of the 18th International Symposium on High Performance Distributed Computing, ACM Press, June 2009

[11] C. Vecchiola, S. Pandey, and R. Buyya, High-Performance Cloud Computing: A View of Scientific Applications, Proc. Of the 10th International Symposium on Pervasive Systems, Algorithms and Networks (I-SPAN 2009), Kaohsiung, Taiwan, Dec. 2009.

[12] C. Dumitrescu, and I. Foster. "GangSim: a simulator for grid scheduling studies," Proc. of the 5th International Symposium on Cluster Computing and the Grid (CCGrid 05), IEEE Computer Society, May 2005.

[13] Rajkumar Buyya, Chee Shin Yeo, Srikumar Venugopal, James Broberg, and Ivona Brandic, Cloud Computing and Emerging IT Platforms: Vision, Hype, and Reality for Delivering Computing as the 5th Utility, Future Generation Computer Systems, Volume no 25, And Number 6, Pages: 599-616, ISSN: 0167-739X, Elsevier Science, Amsterdam, The Netherlands, June 2009. 